\title{
Forward modeling of coronal funnels
}

\author{
T. Aiouaz ${ }^{1,2}$, H. Peter ${ }^{1}$, and R. Keppens ${ }^{3}$ \\ 1 Kiepenheuer Institut für Sonnenphysik (KIS) Schöneckstraße 6, 79104 Freiburg, Germany \\ e-mail: tayeb@kis.uni-freiburg.de \\ 2 Institut d'Astrophysique Spatiale (IAS) CNRS-Université Paris XI, 91405 Orsay Cedex, France \\ 3 FOM Institute for Plasma Physics Rijnhuizen Edisonbaan 143439 MN Nieuwegein, Netherlands
}

Received 12 July 2005 / Accepted 11 September 2005

\section{ABSTRACT}

We propose a forward modeling approach of coronal funnels to investigate the outer layers of the solar atmosphere with respect to their thermodynamical properties and resulting emission line spectra. We investigate the plasma flow out of funnels with a new 2D MHD time dependent model including the solar atmosphere all the way from the chromosphere to the corona. The plasma in the funnel is treated in the single-fluid MHD approximation including radiative losses, anisotropic thermal conduction, and two different parameterized heating functions. We obtain plasma properties (e.g. density, temperature and flow speed) within the funnel for each heating function. From the results of the MHD calculation we derive spectral profiles of a low corona emission line (Ne VIII, $770 \AA$ ). This allows us e.g. to study the Doppler shifts across the funnel. These results indicate a systematic variation of the Doppler shifts in lines formed in the low corona depending on the heating function used. The line shift above the magnetic field concentration in the network is stronger than in the inter-network in both cases. However, for one of the heating functions, the maximum blue-shift (outflow) is not to be found in the very center of the funnel but in the vicinity of the center. This is not the case of the second heating function where the maximum is well aligned with the centre of the funnel. This model directly relates for the first time the form of the heating function to the thermodynamic and spectral properties of the plasma in a funnel.

Key words. Sun - magnetohydrodynamics (MHD) - synthetic spectra - funnel

\section{Introduction}

The simplest picture of the transition region (TR), formed between temperatures of $10^{4} \mathrm{~K}$ and $10^{6} \mathrm{~K}$, is that it is the thermal interface between the hot corona and the cooler chromosphere. Since it is clear from the observed structuring that the magnetic field plays a role, simple energy balance models were modified to include the effect of the magnetic field (Gabriel 1976). Later models (Athay 1981) implicitly assume that the magnetic field within the network is unipolar over supergranular scales. However Dowdy et al. (1986) have argued that the observed fine scale structure of the network consists of mixed magnetic polarities. This led them to propose an alternative magnetic "junkyard" picture of the transition region, consisting of coronal funnels and small cool network loops. To study the properties of coronal funnels and their role in accelerating the solar wind close to the Sun we employ a 2D MHD time-dependent, model, including the solar atmosphere all the way from the chromosphere to the corona. We put special emphasis on the plasma flow out of the coronal funnel. The energetics of the plasma include radiative losses, thermal conduction and two different prescribed heating terms. From the results of the MHD calculation we derive the resulting profiles of low coronal emission lines using the CHIANTI database
(Young et al. 2003). In this paper the case of the Ne VIII (770.4 $\AA$ ) line is examined.

\section{Numerical MHD model}

Since Gabriel (1976) most TR models have incorporated a magnetic field, primary cause of the atmosphere geometry. Hackenberg et al. (2000) presents a 2D magnetostatic model where an analytical definition of the magnetic field builds up the funnel structure above the border of two adjacent supergranules. This model includes for the first time closed and open magnetic field lines.

In our case, the same analytical definition was used to prescribe the initial condition for the magnetic field. However, the region where plasma $\beta$ is high, i.e. where the magnetic field is weak or/and where the plasma pressure is strong, are taken into account, resulting in a dynamic, time dependent, MHD model.

\subsection{Energy equation}

The evolution of the funnel is studied using the conservative form of the MHD equations, including the energy equation,

$\frac{\partial e}{\partial t}+\nabla \cdot\left[\boldsymbol{v}\left(e+p_{\mathrm{th}}+\frac{B^{2}}{2 \mu_{0}}\right)-\frac{(\boldsymbol{v} \cdot \boldsymbol{B}) \boldsymbol{B}}{\mu_{0}}\right]=\boldsymbol{v} \cdot \mathcal{F}+\mathcal{S}$ 
The conservative variables are the density $\rho$, the momentum $\rho \boldsymbol{v}$, the total energy density $e$, and the magnetic field $\boldsymbol{B}$. In the right hand side, $\mathcal{F}$ is a force which represents the effects of gravity and viscosity defined as $\mathcal{F}=\rho \boldsymbol{g}-\nabla \cdot(v \Pi)$ where $\boldsymbol{g}$ is the gravitation field, $v$ the dynamic viscosity given for a fully-ionized $H$ plasma by Spitzer (1962), and $\Pi$ the dynamic pressure tensor. The second term in the right hand side of Eq. (1), $\mathcal{S}$ is describing further sources and sinks, this term is discussed in Sect. 2.3. To solve the MHD equations, we used the Versatile Advection Code (VAC, Tóth 1996) with a non-uniform grid $(100 \times 200)$ where the cells are concentrated in the lower part of the computational domain to resolve the TR.

\subsection{Initial and boundary conditions}

The initial magnetic field is defined analytically (Hackenberg et al. 2000) and is a potential field.

Based on this definition of the magnetic field, we define for the coronal funnel the cross-section $A(z)=B_{\mathrm{u}} / B_{z}(z)$ where $B_{\mathrm{u}}$ is the magnetic field strength at the upper end of the funnel. The density, $\rho$, and the thermal pressure, $p_{\text {th }}$, are initially defined using the hydrostatic pressure balance after that the temperature is prescribed as an hyperbolic tangent. The velocity is set equal to zero everywhere in the domain.

At the lower boundary we keep the normal gradient of density and of the total energy $e$ constant. The momentum is set to zero at the bottom boundary. Thus the low chromosphere artificially acts as a reflective wall for downward propagating waves. In order to keep the funnel structure during the simulation the magnetic field is fixed at the lower boundary.

Since there may be out-going waves that should leave the domain properly without generating spurious reflections at the upper boundary, the top boundary is open. This means that the density, momentum and magnetic field are linearly extrapolated. The total energy at the top is set such that the gradient of the temperature at the top is zero to avoid downward heat flux into the domain. The left and right boundaries are periodic.

\subsection{Energy balance}

In the energy equation (Eq. (1)) the energy sources and sinks, $\mathcal{S}$, represents

$\mathcal{S}=\nabla \cdot \boldsymbol{q}+L_{\mathrm{rad}}+H\left[+C_{\mathrm{N}}\right]$

Here the heat conduction, $\nabla \cdot \boldsymbol{q}$, is along the magnetic field and follows the Spitzer law (Spitzer 1962). The radiative losses, $L_{\mathrm{rad}}$, are assumed to have the tabulated form given by Rosner et al. (1978) for an optically thin medium. Finally to maintain a corona against conductive, radiative, and solar wind losses we specify two different parameterized heating functions, $H_{\exp }(z)$ and $H_{B^{2}}(x, z)$ defined in Eqs. (3) and (4).

The first heating term we implement $H_{\exp }(z)$ varies with height $z$ (vertical direction) only decreasing exponentially in the corona. This form of the heating function is often used in 1D studies of coronal loops (e.g. Serio et al. 1981) and the solar wind (e.g. Hansteen \& Leer 1995). As the density roughly decreases exponentially this function describes a heating process being approximately a power law of the particle density.

$H_{\exp }(z)= \begin{cases}\frac{F}{A(z) \cdot \lambda} & \text { for } z<z_{\mathrm{chr}} \\ \frac{F}{A(z) \cdot \lambda} \exp -\left[\left(\frac{z-z_{\mathrm{chr}}}{\lambda}\right)\right] & \text { for } z>z_{\mathrm{chr}} .\end{cases}$

In Eq. (3) the energy flux into the funnel, $F$, and the damping length scale, $\lambda$, are constants equal to $150 \mathrm{~W} / \mathrm{m}^{2}$ and $3 \mathrm{Mm}$ respectively (Hansteen \& Leer 1995). $z_{\mathrm{chr}}$ is set arbitrarily to $3 \mathrm{Mm}$.

The second heating term $H_{B^{2}}(x, z)$ we implement is proportional to the square of the magnetic field strength, and thus varies in the horizontal $x$ and vertical $z$ direction. It is motivated by recent studies of coronal active regions by Gudiksen $\&$ Nordlund (2002). They show that for braiding of magnetic flux and subsequent dissipation of currents (on average) the heating is roughly proportional to $B^{2}$.

$H_{B^{2}}(x, z)=C \cdot B^{2}(x, z)$.

In Eq. (4) $C$ is a constant which is adjusted such that the total amount of energy over the physical domain is approximately the same for both heating functions.

The last term of the energy sources and sinks in Eq. (2), in brackets, represents the Newton's law of cooling states, $C_{\mathrm{N}}=K\left(\rho / \rho_{1}^{(0)}\right)^{\alpha}\left(T-T^{(0)}\right)$, where $\rho_{1}^{(0)}$ is the density at $t=0$ at the bottom boundary, $T^{(0)}$ is the temperature at $t=0, K$ and $\alpha$ are adjustable constants which controls the area at the bottom boundary where $C_{\mathrm{N}}$ is not negligible $(K=3, \alpha=4)$. This term prevents the chromosphere to completely disappear from the computational domain in case of too high heating. This ensures the numerical stability of the calculation by preserving chromospheric temperature at the bottom boundary. Newton cooling acts only on the few first cells of the computational domain due to the high value of $\alpha$ and to the exponential decrease of the density with $z$ (height).

\section{Thermodynamical properties}

Beginning with hydrostatic pressure balance, we reach a quasi steady state after approximately $35 \mathrm{~min}$ of physical time, which represents approximately six sound travel times. Figures 1 shows a snapshot of the quasi steady solutions for the temperature for both heating functions. It represents the temperature in false color. The arrows indicate the direction of the flow as well as the velocity, which is proportional to the arrow length.

Firstly we can see from Fig. 1 that the TR is not at the same height across the funnel in both cases. Furthermore the temperature in the corona is lower in the centre of the funnel than the surrounding region at the same height. A closer look at the central region, where the TR descends downward in the solar atmosphere, shows that the edges of this central area are the deepest position of the TR.

In the center of the funnel the heat from the corona is conducted deep in the "cold" atmosphere, conduction allowed by the open magnetic field lines. This shows the crucial role played by the magnetic field topology. The conduction moves 

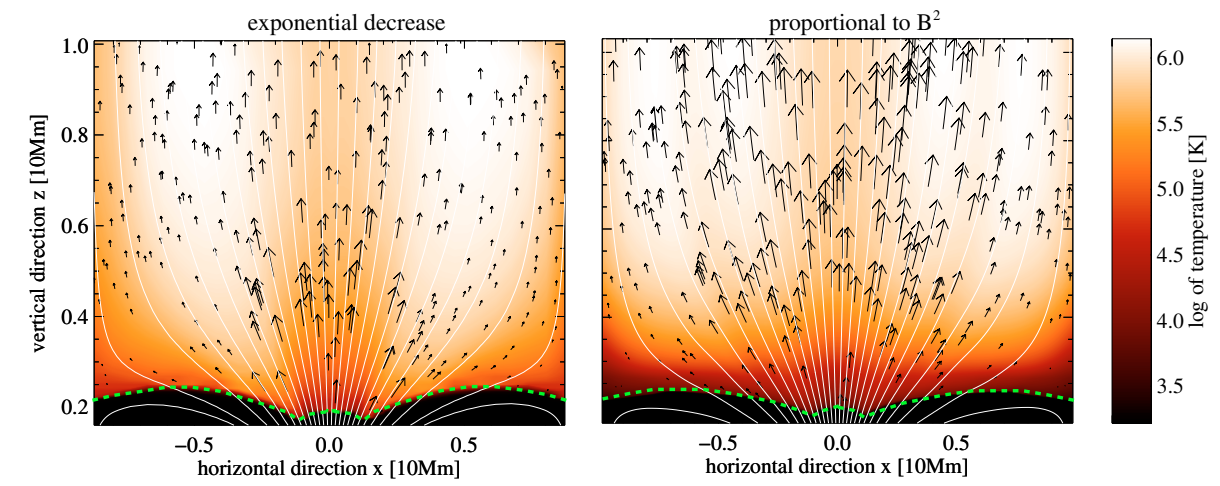

Fig. 1. This figure shows the steady state solution at $t \approx 35 \mathrm{~min}$ of the temperature for the case $H=H_{\exp }(z)$ (left panel) and for $H=H_{B^{2}}(x, z)$ (right panel). The temperature is shown by the filled color contours. The arrows indicate the direction of the flow as well as the velocity, which is proportional to the arrow length. The magnetic field lines are outlined by the white solid lines. The thick dotted line outlines the position of the Transition Region. the TR toward higher densities until it reaches a balance with radiative cooling which is proportional to the square of the density. At the closed magnetic field areas, the topology does not allow any thermal connection between the chromosphere and the corona, and thus does not allow any heat transfer between the closed magnetic field areas and the corona, maintaining then the TR higher up in the atmosphere.

Furthermore we obtain a plasma flow which is stronger toward the center of the funnel. This last characteristic of the funnel is also observed in the solar corona when looking at coronal lines above quiet Sun areas. The internetwork region shows on average less outflow than the network where the magnetic field is concentrated (Aiouaz et al. 2005).

Hammer (1982) established that the variation of the thermal pressure, cause of the velocity variation along the magnetic field lines, is related to the heating. He argues that with increasing heating at the base of the TR the atmosphere is capable of radiating away more energy. Since the radiative losses are coupled to the density and thus to the thermal pressure (at chromospheric temperature), the pressure increases to radiate away the supplementary energy.

However, to be able to compare this result with observations of the Doppler shifts in the upper transition region or low corona, we must find out how a spectrometer would see the coronal funnel and his thermodynamical properties. To answer this question, we developed a method to create synthetic spectra which correspond to what a spectrometer would see from such a funnel. The spectral code is described in the next section.

\section{Spectral code}

At this point we assume ionization equilibrium to calculate the line emission. As our velocities are quite moderate compared to ionization times this is a possible simplification (see Peter et al. 2004). Under this assumption the emissivity of an optically thin spectral line is defined as $\varepsilon_{j i}=h v_{j i} A_{j i} n_{j} \quad\left[\mathrm{~W} \mathrm{~m}^{-3}\right]$, where $i, j$ are the lower and upper levels, $A_{j i}$ is the spontaneous transition probability, $n_{j}$ is the number density of the upper level $j$ of the emitting ion.

The spectral code uses as input, the values of density $\rho$, temperature $T$, and the plasma flow $v_{z}$ along the line of sight $z$, values provided by the 2D MHD quasi steady state solutions. The ion emissivity, $\varepsilon_{j i}$, is computed at each grid point of the computational domain using the CHIANTI database
(Young et al. 2003). Once the ion emissivities are calculated (lower panels in Fig. 2), a normalized gaussian profile is associated at each grid point to calculate emission profiles $\varepsilon_{v}$, defined as

$\varepsilon_{v}=\frac{\varepsilon_{j i}}{\pi^{1 / 2} \Delta v_{\mathrm{D}}} \exp \left[-\frac{v_{z}^{2}}{\Delta v_{\mathrm{D}}^{2}}\right]$.

Here the temperature is used to calculate the Doppler width, $\Delta v_{\mathrm{D}}=\left(2 k T / M_{i}\right)^{1 / 2}$, and the velocity along the line of sight $v_{z}$ to calculate the Doppler shift. $M_{i}$ is the mass of the considered ion. We integrated then the emission line profiles along the vertical direction ( $z$ direction). This gives us total line profiles across the funnel (i.e. horizontally). From these integrated spectra along the line of sight (i.e vertically) we extracted the intensity, the line width and the line shift.

\section{Results for Ne VIII $770.4 \AA$}

The upper panels in Fig. 2 shows the results for the Ne VIII (770.4 $\AA$ ) emission line Doppler shifts. The results indicate a systematic variation of the Doppler shift in this line formed in the low corona depending on the heating function involved. In this study the Ne VIII ions are assumed to have the same speed as the proton-electron background. As found by Lie-Svendsen $\&$ Esser (2005), in a 1D minor ion win model, the minor species velocities can differ significantly from the main gas once the density dropped below some $10^{13} \mathrm{~m}^{-3}$ above some $15 \mathrm{Mm}$ height. In our model, however, Ne VIII is formed at densities of typically $10^{14} \mathrm{~m}^{-3}$ at heights typically around $z=3 \mathrm{Mm}$. Therefore we can expect only a small difference in speed between the Ne VIII ions and the main gas.

Firstly, the absolute values of the plasma flow (around $10 \mathrm{~km} \mathrm{~s}^{-1}$ ) are in agreement with the usual values observed for the quiet Sun with the same emission line (see upper panels in Fig. 2). Secondly, the line shift above the magnetic field concentration in the network is stronger than in the inter-network in both cases. This result shows that the plasma flow computed and discussed in Sect. 3, where the strongest flows are observed toward the centre, would be observed from a spectrometer looking at a low coronal line. Finally, the upper panels in Fig. 2 show that for $H_{\text {exp }}(z)$ (left panel), we find a dip in Doppler shift in the very center of the funnel. So the largest shifts are not to be found in the very center but in the vicinity of the center. While for $H_{B^{2}}(x, z)$ (right panel) this is not the case, the maximum of Doppler shift is well centered. 

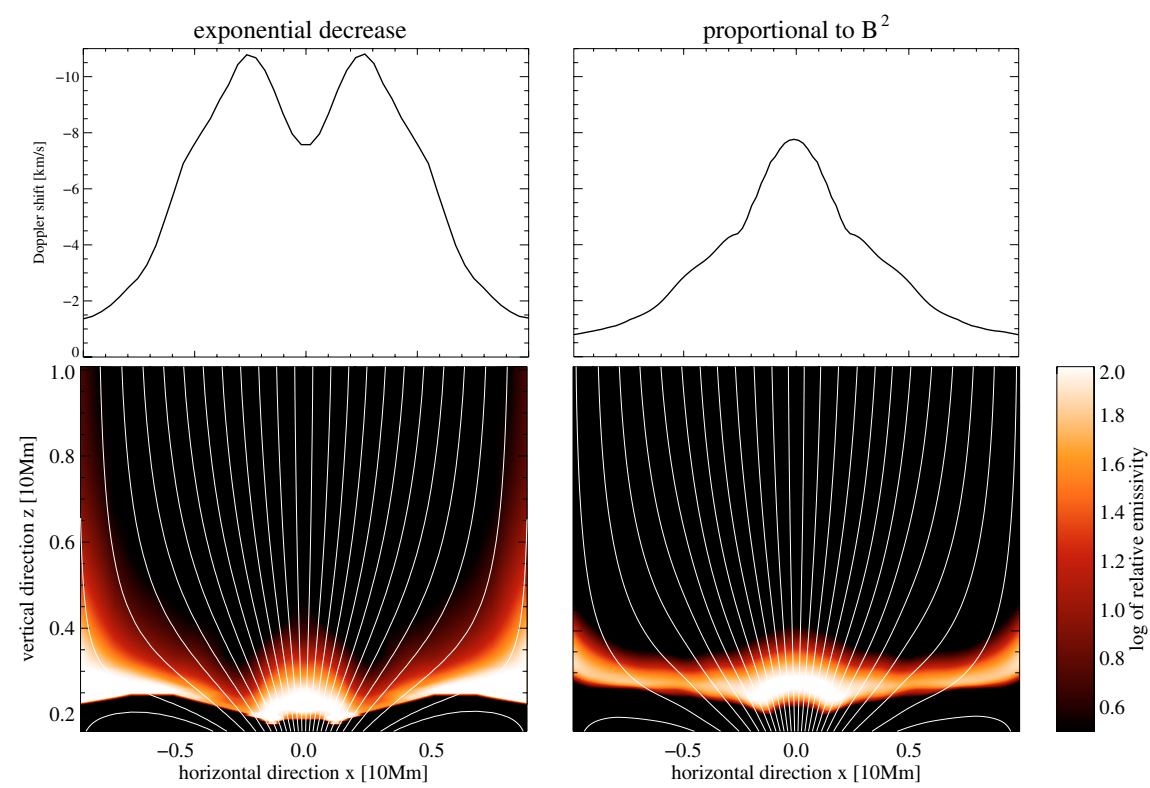

Fig. 2. The upper panels show the line shifts of Ne VIII (770.4 $\AA$ ) line across the funnel calculated for the resulting synthetic spectra integrated along the line of sight (z-direction). The lower panels show the corresponding emissivity, $\varepsilon_{j i}$, of Ne VIII (770.4 $\AA$ ) calculated from the MHD results using the CHIANTI database in the case $H=H_{\text {exp }}(z)$ (left panel) and in the case $H=H_{B^{2}}(x, z)$ (right panel). The contour lines show magnetic field lines.

In the case of $H=H_{\exp }(z)$, since the amount of heating is constant in the horizontal direction (decreases only vertically) then the heating is higher at the base of the TR for the edges of the central part than for the very center. While in the second case $H=H_{B^{2}}(x, z)$, the heating flux decrease vertically and horizontally. So that the win of pressure at the base of the TR for the edges of the central part due to the shift downward in the $z$-axis is balanced by a loss due to the shift in the $x$-axis. This argument explains why the pressure gradient and thus the velocity is stronger at the edges of the central part in the case of exponential decreasing heat $\left(H=H_{\exp }(z)\right)$ and not in the case $H_{B^{2}}(x, z)$ where the heating input is proportional to $B^{2}$. This property is of crucial importance because it directly links the form of the heating function and an observable of the plasma in the funnel, namely the velocity in the upper transition region/or low corona. This last point shows that the Doppler shift in the low corona across coronal funnels is a good candidate for the investigation of the coronal heating mechanism.

\section{Conclusion}

The model presented above is the first dynamic 2D-MHD model of funnels including the calculation of spectral profiles. We are able to quantify the precise variation of the height of the TR across the funnel and compute the flow out for two different heating functions. From the MHD results we used the temperature, density and the velocity of the plasma to compute emissivities using the CHIANTI database. We calculated then synthetic line profiles using the thermodynamic properties and integrated it along the line-of-sight. This gives us total line profiles across the funnel that could be compared to spectroscopic observation with the slit placed across a network lane. This allowed us to study the Doppler shifts across the funnel. These results indicate that the outflow is stronger in the central part of the funnel than at the sides. Furthermore in the middle area of the funnel, we find that the flow strongly depends from one heating function to the other. In the of the case of a heating function decreasing only with height, we find a dip in velocity in the very center of the funnel. So largest Doppler shift in the low corona are not to be found in the very center but in the vicinity of the center. While for heating function proportional to $B^{2}$ this is not the case, the maximum outflow is well centered. This result is of major importance because it gives a diagnostic tool for the investigation of the heating mechanism in the corona. Even though "only" two heating functions were used in this study, it shows how one can relate the heating form to the thermodynamical and spectral properties of the plasma in a funnel. This study motivates further work on funnel modeling and as well as on network observations. Recently some works (see Xia et al. 2003; Aiouaz et al. 2005) have tried to relate those components of the solar atmosphere our understanding of the coronal heating and the structure of the solar atmosphere.

\section{References}

Aiouaz, T., Peter, H., \& Lemaire, P. 2005, A\&A, 435, 713

Athay, R. G. 1981, ApJ, 249, 340

Dowdy, J. F. J., Rabin, D., \& Moore, R. L. 1986, Sol. Phys., 105, 35

Gabriel, A. H. 1976, Phil. Trans. R. Soc. Lond. A., 281, 339

Gudiksen, B. V., \& Nordlund, Å. 2002, ApJ, 572, L113

Hackenberg, P., Marsch, E., \& Mann, G. 2000, A\&A, 360, 1139

Hammer, R. 1982, ApJ, 259, 779

Hansteen, V. H., \& Leer, E. 1995, JGR, 100, 21577

Lie-Svendsen, Ø., \& Esser, R. 2005, ApJ, 618, 1057

Peter, H., Gudiksen, B. V., \& Nordlund, A. 2004, ApJ, 617, L85

Rosner, R., Tucker, W. H., \& Vaiana, G. S. 1978, ApJ, 220, 643

Serio, S., Peres, G., Vaiana, G. S., Golub, L., \& Rosner, R. 1981, ApJ, 243, 288

Spitzer, L. 1962, Physics of Fully Ionized Gases (New York: Interscience) (2nd edition)

Tóth, G. 1996, Astrophys. Lett., 34, 245

Xia, L. D., Marsch, E., \& Curdt, W. 2003, A\&A, 399, L5

Young, P. R., Del Zanna, G., Landi, E., et al. 2003, ApJ, 144, 135 